\title{
Research on multifractal dimension and improved gray relation theory for intelligent satellite signal recognition
}

\author{
Zhen Zhang ${ }^{1} \cdot$ Yibing $\mathrm{Li}^{1} \cdot \mathrm{Lin}^{\mathrm{Qi}}{ }^{1}$
}

(C) The Author(s) 2019

\begin{abstract}
In the wake of the development and advancement of signal processing technology for communication radiation source individual, Signal fingerprint feature extraction and analysis technology for communication radiation source individual has broad application prospects in many fields. To effectively extract the individual characteristics of different modulated signals under low SNR environment, and recognize the subtle features for communication radiation source individuals has been a hot spot. Aiming at the problem of signal feature extraction and classifier design under low SNR environment, in the paper, a multifractal dimension and improved gray relation theory based classifier design algorithm is proposed. Firstly, the multifractal dimension feature extraction of nine modulated communication signals is realized. Then multifractal dimension features of these modulated signals under different SNR are compared. An improved gray relation algorithm is used to recognize the extracted subtle characteristics. Meanwhile, FSK signal is used to simulate radio subtle features by adding different distribution of noise. Subtle feature extraction by means of multifractal dimension algorithm and pattern recognition by means of improved gray relation algorithm are used to test the effectiveness of the proposed method for identification of modulated signals and radio subtle features. The simulation results show that the recognition success rate of nine different communication modulated signals can reach 93\% even under the SNR of $2 \mathrm{~dB}$, and the recognition success rate of the subtle features of distributed noise can reach 100\%. The proposed method provides an effective theoretical basis for identifying of radio modulated signals and communication radiation source individual subtle features.
\end{abstract}

Keywords Feature extraction - Classifier design · Intelligent satellite signal · Multifractal dimension · Improved interval gray relation theory

\section{Introduction}

Under the background of the increasingly widespread application of communication technology disciplines and the rapid development of signal processing disciplines, the identification of communication radiation source individuals has gradually attracted the researchers attention [1,2]. With the continuous improvement of anti-reconnaissance and anti-jamming technologies, traditional intelligence information methods are difficult to achieve the information acquisition. With the advance in technologies such as

Lin Qi

qilin@hrbeu.edu.cn

1 College of Information and Communication Engineering, Harbin Engineering University, Harbin 150001, People's Republic of China computer technology, fusion algorithms, and electronic information science, signal feature analysis [3] and signal information extraction techniques [4] also develop rapidly, thus achieving the full use of information that was not available in the past. The contained target information from the signal characteristics and subtle features of communication radiation source individuals has good mining potential $[5,6]$. How to use the subtle features of the radiation source individuals to achieve reconnaissance and surveillance of targets on the battlefield in today's complex electromagnetic environment is a new and valuable research field [7, 8]. This technology is deeply welcomed by various combat troops and has great application value. The fractal theory [9-12] is often referred to as the geometry that exists in nature and complements the chaos theory of the dynamic system. In 1975, the American French mathematician, B.B. Mandelbrot, first proposed the 
geometric concept of fractals. With the continuous development of fractal theory, he pointed out that parts of anything in the world may be in a certain process, or under certain conditions, certain aspects of things (such as energy, function, information, time, structure, morphology, etc.) show similarities with the whole. And the change in spatial dimension can be continuous or discrete, which expands people's horizons.

Fractal dimension theory has been widely used in fault diagnosis [10, 13], image information processing [14, 15] and other fields, which can effectively describe the waveform characteristics of signals. For instance, the fractal-box dimension algorithm uses the boxes with different side lengths to depict the signal waveform change [16, 17]. Literature [18] uses the box dimension algorithm to depict the signal features, where the smaller the box side length, the longer the computational time, the higher the recognition success rate of signals. The multifractal dimension algorithm can describe the complexity and irregularity of the signal compared with fractal-box dimension algorithm [19]. It can calculate different dimension characteristics of signals, describe signal features from different dimensions, and reflect the distribution density of signal in the area space, thereby achieving recognition of the signal. Ding Kai et al. used the multifractal dimension algorithm to identify different signals, and the high recognition efficiency was achieved in the lower SNR environment, but the proposed algorithm has high computational complexity and costs long simulation time [20]. Yang et al. applied the fractal theory to the analysis and processing of noise, which has a wide range of application effects. It mainly discusses the root cause, model and analysis method of $1 /$ f noise, and the application of fractal estimation in signal processing is well summarized [21]. Wu et al. studied the fractal growth model and summarized its research in materials science [22]. The literature [23-25] summarizes the application of fractals in heat transfer and mass transfer of media, the application in geomorphology, and the application in manufacturing systems.

It can be seen from the literatures in recent years that fractal dimension has achieved good development in various fields. Therefore, the research on fractal signal recognition [26-28] has also become the focus of this paper. The main contributions of this paper are as follows: first, extract the multifractal dimension features of nine modulated signals under different SNR environment, then the features of FSK signals with different distributed noise are extracted, at last, an improved adaptive interval gray relation algorithm $[29,30]$ is used to classify the extracted characteristics, and finally the purpose of accurately identifying signals is achieved.

\section{Basic definition of multifractal dimension}

Multifractal dimension algorithm can depict the signals features from different levels and describe the signal waveform probability distribution characteristics. Multifractal dimension algorithm splits the research object into $N$ small regions. Set the linearity of $i$ th region as $\varepsilon_{i}$, and the density distribution function $P_{i}$ of $i$ th region can be described by different scale indices $\alpha_{i}$ as following:

$P_{i}=\varepsilon_{i}^{\alpha_{i}}, \quad i=1,2, \ldots, N_{i}$

The non-integer $\alpha_{i}$ is generally called a singularity index, and its value corresponds to its region.

To obtain the distributed characteristics of a succession of subsets, define the function $X_{q}(\varepsilon)$, which is the probability-weighted summation of the regions:

$X_{q}(\varepsilon)=\sum_{i=1}^{N} P_{i}^{q}$

Further the generalized fractal dimension $D_{q}$ can be obtained as follows:

$D_{q}=\frac{1}{q-1} \lim _{\varepsilon \rightarrow 0} \frac{\ln X_{q}(\varepsilon)}{\ln \varepsilon}=\frac{1}{q-1} \lim _{\varepsilon \rightarrow 0} \frac{\ln \left(\sum_{i=1}^{N} P_{i}^{q}\right)}{\ln \varepsilon}$

$X_{q}(\varepsilon)$ shows the role of various sizes $P_{i}$, and it can be seen from the Eq. (3) that, the area with high probability plays a major role of the sum $\sum P_{i}^{q}$ when $q \gg 1$, and at this time, $X_{q}(\varepsilon)$ and $D_{q}$ reflect the nature of the high probability region (i.e., dense region)When $q \rightarrow \infty$, the small probability can be ignored, and only the large probability $P_{i}$ is considered, so that the calculation of $D_{q}$ is simplified. On the contrary, $X_{q}(\varepsilon)$ and $D_{q}$ reflect the nature of a small probability region (i.e., sparse region) when $q \ll 1$. Thus, the properties of different probability characteristic regions are represented by different $q$ values. After the weighted summation process, a signal is divided into a number of regions, which have different degrees of singularity. Therefore, it is possible to understand the fine structure inside the signal hierarchically.

Define $D_{q}$ as capacity dimension (i.e., box dimension) $D_{0}$, information dimension $D_{1}$, and correlation dimension $D_{2}$ respectively when $q=0,1,2$.

\section{Design method of classifier based on gray relation}

\subsection{Ordinary gray relation algorithm}

The basic idea of gray relation theory is to quantitatively describe and compare the changes and development trends 
of a system. Suppose the behavior sequence of the system is:

$$
\begin{gathered}
X_{0}=\left(x_{0}(1), x_{0}(2), \ldots, x_{0}(n)\right) \\
X_{1}=\left(x_{1}(1), x_{1}(2), \ldots, x_{1}(n)\right) \\
\ldots \\
X_{i}=\left(x_{i}(1), x_{i}(2), \ldots, x_{i}(n)\right) \\
\ldots \\
X_{m}=\left(x_{m}(1), x_{m}(2), \ldots, x_{m}(n)\right)
\end{gathered}
$$

where $X_{0}$ represents the reference sequence and $X_{1}, X_{2}, \ldots, X_{m}$ represents the comparative sequence.

Set:

$$
\begin{aligned}
& \gamma\left(x_{0}(k), x_{i}(k)\right) \\
& =\frac{\min _{i} \min _{k}\left|x_{0}(k)-x_{i}(k)\right|+\rho \max _{i} \max _{k}\left|x_{0}(k)-x_{i}(k)\right|}{\left|x_{0}(k)-x_{i}(k)\right|+\rho \max _{i} \max _{k}\left|x_{0}(k)-x_{i}(k)\right|} \\
& \gamma\left(X_{0}, X_{i}\right)=\frac{1}{n} \sum_{k=1}^{n} \gamma\left(x_{0}(k), x_{i}(k)\right)
\end{aligned}
$$

Here, define $\rho \in(0,1)$ as the resolution coefficient, which is usually set as 0.5 . And $\gamma\left(X_{0}, X_{i}\right)$ is called the gray relation degree between $X_{0}$ and $X_{i}$, often abbreviated as $\gamma_{0 i}$. The k-point relation coefficient $\gamma\left(x_{0}(k), x_{i}(k)\right)$ is abbreviated as $\gamma_{0 i}(k)$.

The specific calculation process of the gray relation degree is listed as follows:

Step 1: Calculate the initial phase value (or mean value phase) for each sequence, that is:

$$
X_{i}^{\prime}=\frac{X_{i}}{x_{i}(1)}=\left(x_{i}^{\prime}(1), x_{i}^{\prime}(2), \ldots, x_{i}^{\prime}(n)\right), \quad i=0,1,2, \ldots, m
$$

Step 2: Calculate the difference sequences, that is:

$$
\begin{aligned}
& \Delta x_{i}(k)=x_{0}^{\prime}(k)-x_{i}^{\prime}(k), \Delta x_{i}= \\
& \left(\Delta x_{i}(1), \Delta x_{i}(2), \ldots, \Delta x_{i}(n)\right), \quad i=1,2, \ldots, m
\end{aligned}
$$

Step 3: Calculate the maximum difference and the minimum difference, namely:

$M=\max _{i} \max _{k} \Delta x_{i}(k), m=\min _{i} \min _{k} \Delta x_{i}(k)$

Step 4: Calculate the relation coefficient value as follows:

$\gamma_{0 i}(k)=\frac{m+\rho M}{\Delta x_{i}(k)+\rho M}, \rho \in(0,1), k=1,2, \ldots, n ;$

$i=1,2, \ldots, m$

Here, $\rho$ is named as resolution coefficient, which values between 0 and 1 .
Step 5: Finally, calculate the relation value between sequences, namely:

$$
\gamma_{0 i}=\frac{1}{n} \sum_{k=1}^{n} \gamma_{0 i}(k), i-1,2, \ldots, m
$$

Then $\gamma_{0 i}$ represents the relation degree between the sequences, that is, the similarity degree between the sequences, $n$ is the number of features.

\subsection{Interval gray relation algorithm}

The interval gray relation algorithm uses the feature interval of the signals to classify the extracted features, and has better recognition effect for overlapping features. The calculation process is depicted as follows:

Define the feature interval matrix first as:

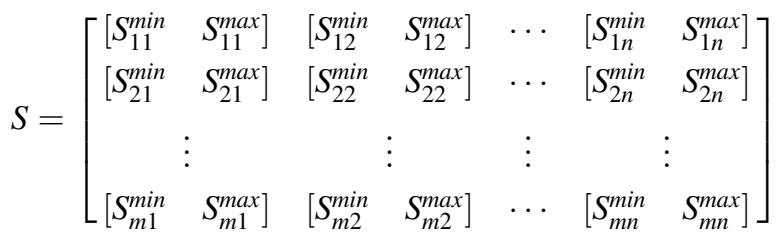

where $m$ represents the signal type; $n$ represents the number of characteristic parameters, and $s_{m n}^{\min }$ represents the minimum value of the fluctuation range of the $n$th eigenvalue of the modulated signal $m ; s_{m n}^{\max }$ represents the maximum value of the fluctuation range of the nth eigenvalue of the modulation signal $m$.

Suppose the feature interval of the $n$th feature of the signal to be identified is $\left[\begin{array}{ll}S_{0 n}^{\min } & S_{0 n}^{\max }\end{array}\right]$, then the interval dissociation degree of the known signal feature interval is defined as:

$$
d_{\text {min }}=\frac{1}{\sqrt{2}} \sqrt{\left(s_{\text {mnmin }}-s_{0 n \min }\right)^{2}+\left(s_{\text {mnmax }}-s_{0 n \max }\right)^{2}}
$$

Then the interval gray relation coefficient can be obtained as follows:

$$
\xi_{m n}=\frac{\min _{m} \min _{n}\left\{d_{m n}\right\}+\rho \max _{m} \max _{n}\left\{d_{m n}\right\}}{d_{m n}+\rho \max _{m} \max _{n}\left\{d_{m n}\right\}}
$$

where $\rho=0.5$ By calculating the value of $\xi_{m n}$ in order, the interval relation coefficient matrix can be constructed:

$\left[\xi_{i j}\right]=\left[\begin{array}{cccc}\xi_{11} & \xi_{12} & \cdots & \xi_{1 n} \\ \xi_{21} & \xi_{22} & \cdots & \xi_{2 n} \\ \vdots & \vdots & \vdots & \vdots \\ \xi_{m 1} & \xi_{m 2} & \cdots & \xi_{m n}\end{array}\right]$

where $i=1,2, \ldots, m, \quad j=1,2, \ldots, n$. 
Therefore the gray relation degree can be calculated as follows:

$$
\xi_{i}=\frac{1}{n} \sum_{j=1}^{n} \xi_{i j}
$$

The value of $i$ corresponding to the largest $\xi_{i}$ value is the category to which the signal to be identified belongs.

\subsection{Improved adaptive mean gray relation algorithm}

Suppose there is a certain feature parameter $x$ from feature parameter set $X$ of a certain signal and the value of its possible eigenvalue is $\left\{x_{i}\right\}=\left\{x_{1}, x_{2}, \ldots, x_{n}\right\}$. And for any $i, j, x_{i} \neq x_{j}$, take the mean value of the $n$ number of eigenvalues as the standard reference value:

$x_{0}=\frac{x_{1}+x_{2}+\cdots+x_{n}}{n}$

Theoretical analysis shows that the mean value of eigenvalues is selected as the reference sequence, which has better stability compared with the randomly selected reference samples. When the mean value samples are close, that is, the parameter values of different signals overlap too much, the recognition success rate will drop rapidly, so the inter-class distances between samples are required by the improved algorithm to some extent.

For the improved adaptive mean value sample relation algorithm, considering with the concept of residual in information theory, the feature distance $\Delta x_{i}(k)$ is processed as follows:

$p_{i}(k)=\Delta x_{i}(k) / \sum_{i=1}^{m} \Delta x_{i}(k)$

where $m$ is the type of signal and the expression of the entropy is:

$$
E_{k}=-\sum_{i=1}^{m} p_{i}(k) \ln p_{i}(k)
$$

Then the maximum entropy expression is:

$$
E_{\text {max }}=-\sum_{i=1}^{m} p_{i}(k) \ln p_{i}(k)=-\sum_{i=1}^{m} \frac{1}{m} \ln \frac{1}{m}=\ln m
$$

Then the relative entropy value is:

$$
e_{k}=E_{k} / E_{\max }
$$

In view of the concept of residual in information theory, the residual of the $k$ th eigenvalue is:

$$
D_{k}=1-e_{k}
$$

The substantial meaning of the residual is the removal of the difference between the $k$ th characteristic entropy value and the optimal feature entropy value. The larger $D_{k}$ is, the more important the feature is, and the greater the weight should be given. Finally, the weight $a_{i k}$ of the $k$ th eigenvalue is calculated as:

$a_{i k}=D_{k} / \sum_{k=1}^{n} D_{k}$

where $\sum_{k=1}^{n} a_{i k}=1, a_{i k} \geq 0$

Multiply the weight coefficient by the corresponding relation coefficient to obtain the relation degree value, namely:

$\gamma_{0 i}=\sum_{k=1}^{n} a_{i k} \gamma_{0 i}(j), \quad i=1,2, \ldots, m$

From the statistical perspective, the more the deviation, the more the characteristics can reflect the differences between the categories. Therefore, it can be considered that the greater the degree of difference in the features, the more important the feature is. Therefore, Compared with other classifier algorithm [3, 31, 32], the improved gray relation algorithm has a certain adaptive effect and easy to calculate the recognition results.

\subsection{Improved adaptive interval gray relation algorithm}

The improved interval gray relation value algorithm first needs to calculate the relation coefficient matrix $\left[\xi_{i j}\right]$

$\left[\xi_{i j}\right]=\left[\begin{array}{cccc}\xi_{11} & \xi_{12} & \cdots & \xi_{1 n} \\ \xi_{21} & \xi_{22} & \cdots & \xi_{2 n} \\ \vdots & \vdots & \vdots & \vdots \\ \xi_{m 1} & \xi_{m 2} & \cdots & \xi_{m n}\end{array}\right]$

The algorithm of the weight value $a_{i j}$ is the same as the calculation method in Sect. 3.3. Finally, the weight value assigned to the common interval relation algorithm correspondingly is as follows:

$$
\xi_{i}=\sum_{j=1}^{n} a_{i j} \xi_{i j}
$$

The obtained interval gray relation degree $\xi_{i}$ at this time has certain adaptive ability, which has better recognition effect compared with ordinary interval gray relation algorithm.

According to the above discussion, the improved adaptive interval gray relation algorithm was used as a classifier to achieve accurate identification. 


\section{Basic steps of algorithm implementation}

In this section, a new modulation signal feature extraction algorithm based on complexity features is proposed. And multifractal dimension theory is applied to the modulation signal feature extraction. The specific steps of algorithm implementation are as follows:

Step 1: the received unknown communication signal is pre-processed, that is, discretized it as:

suppose the received radiation source individual signal is $S$, and the preprocessed discrete signal sequence be $\{s(i)\}$, where $i=1,2, \ldots, N_{0}$ is the number of signal sampling points and $N_{0}$ is the signal sequence length.

Step 2: reorganize the discretized signal sequence:

First, for the preprocessed discrete communication signal sequence $\{s(i)\}, i=1,2, \ldots, N_{0}$, define the characteristic parameters as follows:

Define: $n=\log _{2}^{N_{0}}$, which indicate the times of the number of different vectors of the signal that to be reorganized.

Define: $t(j)=2^{j}$, which indicates the number of discrete signal points in each recombination signal, where $j=1,2, \ldots, n$ represent the values of the number of different vectors of the recombination signal.

Define a sequence of numbers:

$T(j)=\frac{N_{0}}{t(j)}=\frac{N_{0}}{2^{j}}$

where $j=1,2, \ldots, n$.

Then define the recombination signal sequence :

$S(j)=s\left(T(j) *(t(j)-1)+T_{0}(j)\right)$

where $T_{0}(j)=[1: T(j)], j=1,2, \ldots, n$.

Step 3: the multifractal dimension operations are performed on the reconstructed eigenvectors, and different dimensions are selected to extract the signals multifractal dimension features:

The multifractal dimension feature describes the different level characteristics of things. A multifractal dimension can be regarded as a union of fractal subsets of different dimensions. The research object is divided into $M$ small regions, and set the linearity of the $i$ th region as $\varepsilon_{i}$ and the density distribution function of the $i$ th region as $P_{i}$, and then the scale index $\alpha_{1}$ of the $i$ th region can be described as following.

$P_{i}=\varepsilon_{i}^{\alpha_{i}}, \quad i=1,2, \ldots, N_{i}$

The non-integer $\alpha_{i}$ is called the singular exponent, which represents the fractal dimension of a certain region. Since a signal can be divided into many different small regions, a variable $f(\alpha)$ composed of a series of different $\alpha_{i}$ can be obtained, and $f(\alpha)$ becomes a signal multifractal spectrum.

Define the function $X_{q}(\varepsilon)$ as the weighted summation of the probabilities for each region, and $\varepsilon$ is the magnitude of the linearity, and $q$ is power for density distribution function $P_{i}$, i.e.:

$$
X_{q}(\varepsilon)=\sum_{i=1}^{N} P_{i}^{q}
$$

Define generalized fractal dimension $D_{q}$ as follows:

$$
D_{q}=\frac{1}{q-1} \lim _{\varepsilon \rightarrow 0} \frac{\ln X_{q}(\varepsilon)}{\ln \varepsilon}=\frac{1}{q-1} \lim _{\varepsilon \rightarrow 0} \frac{\ln \left(\sum_{i=1}^{N} P_{i}^{q}\right)}{\ln \varepsilon}
$$

Thus each of the recombination signals $S(j)$ in step 2 is summarized, and $S(j)$ represents the $j$ th recombination signal, namely:

$$
\begin{aligned}
S_{j} & =\sum S(j) \\
& =\sum s\left(T(j) *(t(j)-1)+T_{0}(j)\right) \\
& =\sum_{T_{0}(j)=1}^{T(j)} s\left(T(j) *(t(j)-1)+T_{0}(j)\right)
\end{aligned}
$$

where $J_{1}, 2, \ldots J_{0}, j=1,2, \ldots n, S_{J}$ is the sum of $J$ th recombination signal, and $J_{0}$ is the number of recombination signals.

Then sum the entire discrete signal sequence, and the sum is $S$, ie:

$S=\sum_{i=1}^{N_{0}} s(i)$

where $i=0,1,2, \ldots N_{0}$

$S_{i}$ is $i$ th sample point value for the discrete signal sequence, and then the $J$ th probability measure $P_{j}$ is defined as:

$P_{J}=\frac{S_{J}}{S}$

where $J=1,2, \ldots J_{0}$

The multifractal dimension feature of the signal can be obtained by taking $P_{J}$ into the calculation formula of multifractal dimension $D_{q}$.

Finally, the adaptive interval gray relation algorithm is used to recognize the obtained features, and then the recognition rate is calculated to test the effectiveness of the proposed algorithm. 


\section{Simulation results and analysis}

Take nine commonly used communication modulation signals, such as 16QAM, 2ASK, 2FSK, 4FSK, QPSK, 8FSK, 4ASK, BPSK, 32QAM, calculate the multifractal dimension features under three different SNR environments of $-10 \mathrm{~dB}, 0 \mathrm{~dB}$ and $10 \mathrm{~dB}$. And the simulation results are shown from Figs. 1, 2 and 3, where the abscissa represents Ine and the ordinate represents Inxq respectively, corresponding to two parameters in the definition of multifractal dimension, and each point on the curve represents a set of multifractal feature values for different signals.

It can be seen from the simulation diagram that the multifractal dimension of different modulation signals is obviously different. The signal can be distinguished by the fractal dimension features at different dimensions even at a lower SNR. The gray relation classifier algorithm and its proposed improved algorithm are used to classify and identify the extracted features by using the multifractal features of the signal as feature database. At the same time, the recognition results of the traditional neural network classifier are used to compare and contrast. And the recognition rate under different SNRs is seen in Table 1.

It can be seen from the recognition results in Table 1 that under the SNR of $10 \mathrm{~dB}$ and $20 \mathrm{~dB}$, the four classifiers all have good recognition performance. According to the analysis of Fig. 1, these classifiers can obtain a recognition success rate of more than $95 \%$ when these characteristic parameters have better separation characteristics. And the recognition success rates of the common gray relation algorithm, the improved mean sample relation algorithm, and the improved adaptive mean sample relation algorithm all will decrease when the SNR decreases. Only the neural
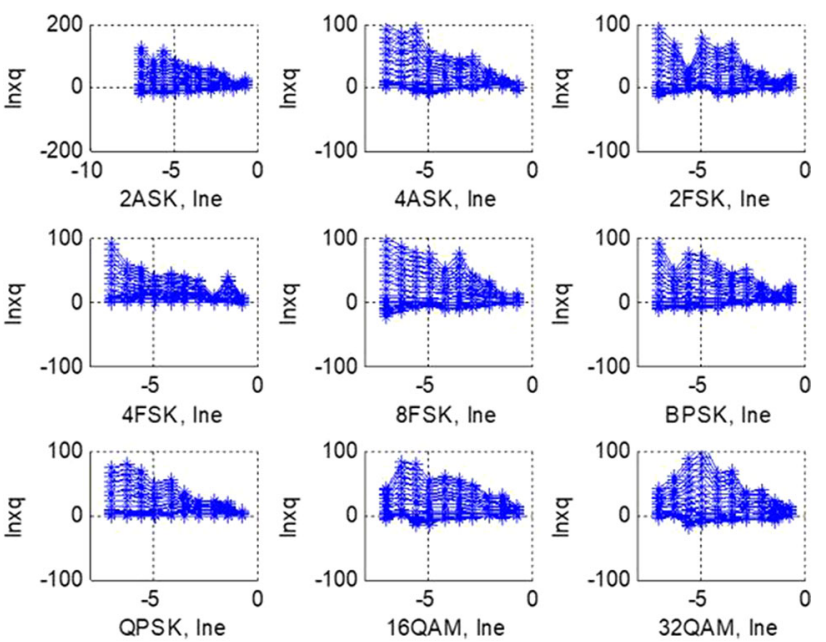

Fig. 1 Multifractal dimension characteristics of different modulated signals under SNR of $10 \mathrm{~dB}$
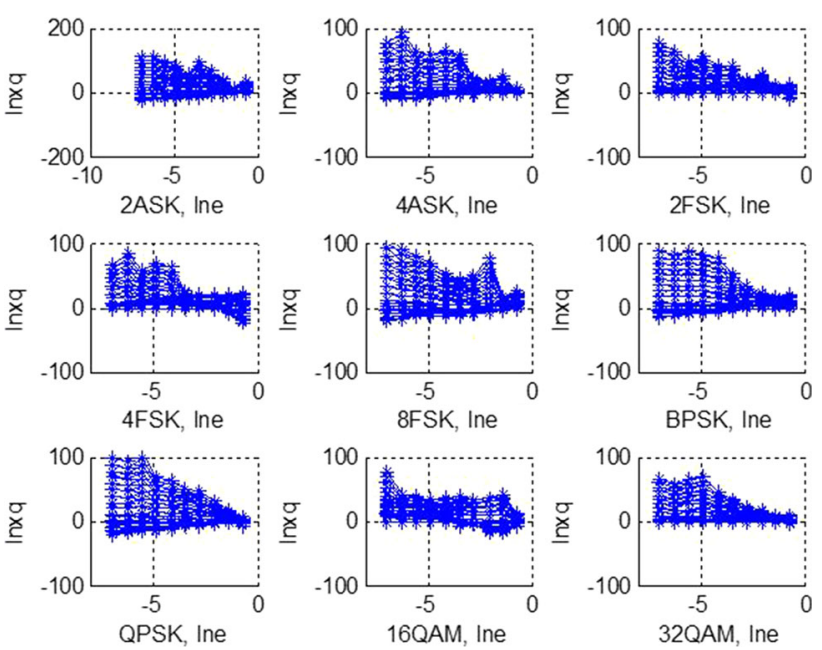

Fig. 2 Multifractal dimension characteristics of different modulated signals under SNR of $0 \mathrm{~dB}$
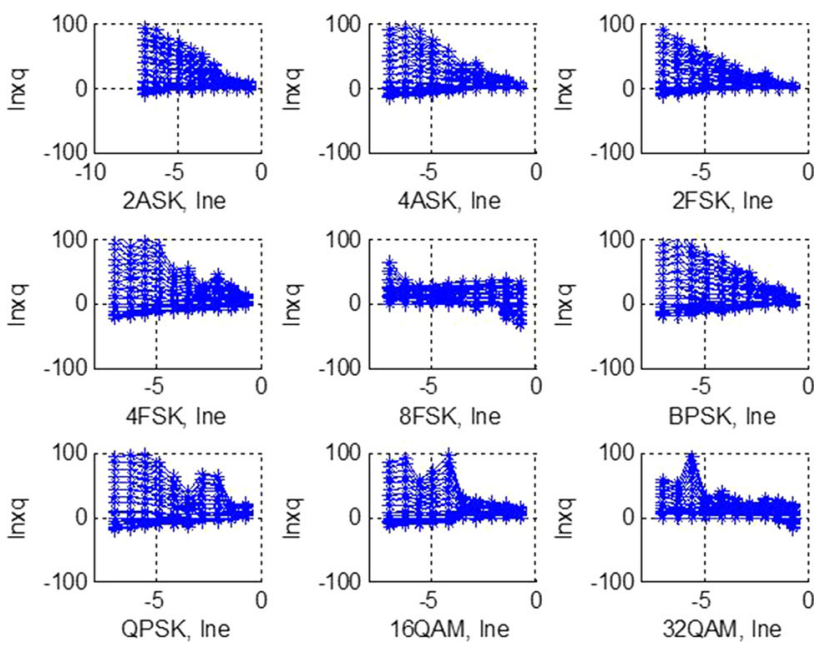

Fig. 3 Multifractal dimension characteristics of different modulated signals under SNR of $-10 \mathrm{~dB}$

network classifier and the gray relation classifier with improved adaptive entropy weight can obtain more than $90 \%$ recognition rate when the SNR is $2 \mathrm{~dB}$. when the SNR is $0 \mathrm{~dB}$, the performance of classifiers obviously decreases. It can be seen from the analysis of Fig. 2 that the neural network based classifier and the entropy weight based interval gray relation algorithm have the best recognition effect when the signal characteristic parameters overlap partially.

In order to measure the complex characteristics for different algorithms, the computational time for different classifiers is shown in Table 2.

It can be seen from the simulation results in Table 2 that the computational time of the gray relation algorithm based classifiers is basically no difference, i.e., the complexity of these classifiers is equivalent. Compared with the improved 
Table 1 The recognition rate of multifractal dimension features by different classifiers under different SNR

\begin{tabular}{llllll}
\hline & $20 \mathrm{~dB}$ & $10 \mathrm{~dB}$ & $5 \mathrm{~dB}$ & $2 \mathrm{~dB}$ & $0 \mathrm{~dB}$ \\
\hline Neural network classifier (\%) & 100 & 100 & 98.6 & 93.2 & 89.6 \\
Ordinary gray relation algorithm (\%) & 100 & 96.5 & 89.2 & 79.6 & 68.6 \\
Improved adaptive mean value sample relation algorithm (\%) & 100 & 98.5 & 90.3 & 82.7 & 76.7 \\
Interval gray relation algorithm cased on entropy weight (\%) & 100 & 100 & 98.5 & 93.0 & 88.7 \\
\hline
\end{tabular}

Table 2 Computational time for different classifiers

\begin{tabular}{lllll}
\hline Algorithm & Neural networks & Gray elation & Improved adaptive mean relation & Interval relation of entropy weight \\
\hline Simulation time (s) & 8.06 & 1.03 & 1.04 & 1.04 \\
\hline
\end{tabular}

adaptive interval gray relation algorithm, the neural network classifier has a better classification effect. However, due to the long time required to train test samples, the improved gray correlation algorithm has better application value than neural network classifier in real-time performance.

Similarly, the FSK signal transmitted by the communication station is taken as an example, and five different distributed noise sequences are generated and added on the FSK signal. Each distribution randomly generates 1000 samples. The Monte Carlo simulation experiment is carried out to explore the recognition effect for different distributed noise. The multifractal characteristic diagram of the FSK signal with different distributed noise sequences and the simulation results are shown in Fig. 4 (where the ordinate A represents the amplitude value; the abscissa represents the time on the left side; the abscissa represents Ine and the ordinate represents $\mathrm{InXq}$ on the right side).

Different distributed noises represent different subtle features carried by radios transmitting of the same signal to test the recognition effect of the proposed method on the subtle "fingerprint" features. It can be seen from the
Fig. 4 FSK signal with different distributed noise sequence and its multifractal dimension curves

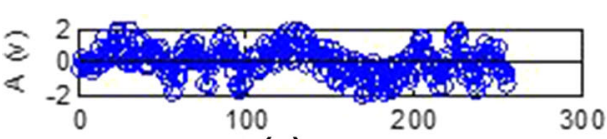

(a) $\mathrm{t}(\mathrm{ms})$

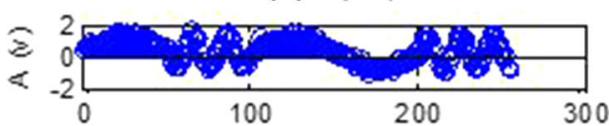

(b) $\mathrm{t}$ (ms)

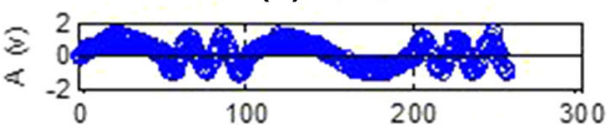

(c) $\mathrm{t}(\mathrm{ms})$

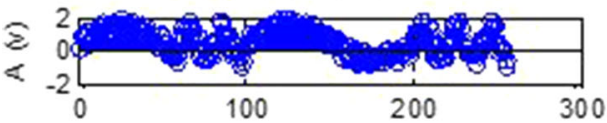

(d) $\mathrm{t}$ (ms)

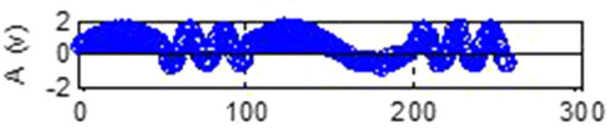

(e) $\mathrm{t}$ (ms)

(a) FSK signal + evenly distributed noise noise

(c) FSK signal + exponential distribution noise noise

(e) FSK signal + normal distribution noise

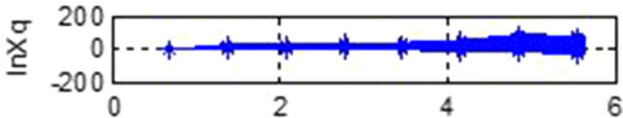

Ine

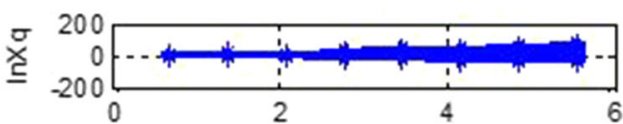

Ine

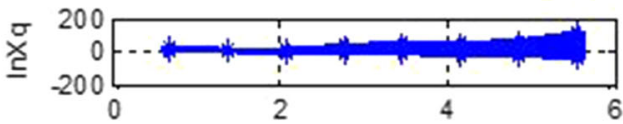

Ine

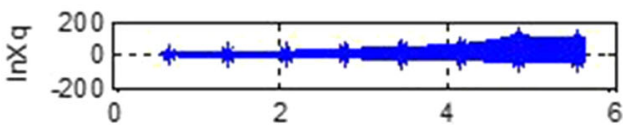

Ine

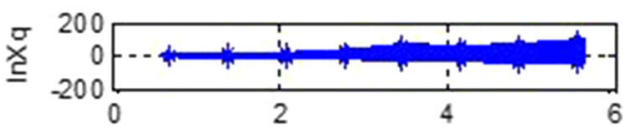

Ine (b) FSK signal + Rayleigh distribution

(d) FSK signal + beta distribution 
simulation results that the multifractal dimension characteristic curves of FSK signal with different distributed noise sequences are slightly different. Using the improved gray relation theory, the relation degree of the fractal results obtained in each reconstruction space are calculated. And the recognition success rate can reach $100 \%$, which verifies the effectiveness of the improved algorithm proposed in the paper.

\section{Conclusion}

After years of development, the individual identification of radiation sources has achieved certain results. But it has not yet formed a complete scientific system, which involves many complicated factors, including the increasing number and complexity of radio signals, the increasing electromagnetic environment, the gradually increasing noise interference, and the application of a large number of new system communication devices, resulting in traditional algorithms in many cases can not accurately achieve signal recognition in complex electromagnetic environments. Aiming at these problems, this paper proposes a multifractal dimension and improved gray relation theory based classifier design algorithm. Firstly, the effectiveness of the proposed algorithm for different types of modulated signals is validated. Then, different radio noises are simulated to validate the effectiveness of subtle feature recognition of signals by the proposed algorithm. Simulation results illustrate that the recognition success rate for nine different communication modulated signals can achieve 93\% recognition rate even under the SNR of $2 \mathrm{~dB}$. And the identification of subtle features of distributed noise can reach $100 \%$ recognition rate. This provides an effective theoretical basis for the identification of radio modulated signals and subtle features of signals. Although the simulation results show the effectiveness of the proposed algorithm, for increasingly complex electromagnetic environment, the radio subtle features are not stable due to many external factors. How to extract robust radio features for complex electromagnetic environments is the hotspot that scholars still need to continue to study in the future.

Acknowledgements This work is supported by the National Natural Science Foundation of China (61771154) and the Fundamental Research Funds for the Central Universities (HEUCFG201830).

Open Access This article is distributed under the terms of the Creative Commons Attribution 4.0 International License (http://creative commons.org/licenses/by/4.0/), which permits unrestricted use, distribution, and reproduction in any medium, provided you give appropriate credit to the original author(s) and the source, provide a link to the Creative Commons license, and indicate if changes were made.

\section{References}

1. Zhang, Z., Guo, X., \& Lin, Y. (2018). Trust management method of D2D communication based on RF fingerprint identification. IEEE Access, 6, 66082-66087.

2. Tu, Y., Lin, Y., Wang, J., et al. (2018). Semi-supervised learning with generative adversarial networks on digital signal modulation classification. CMC-Computers Materials \& Continua, 55(2), 243-254.

3. Lin, Y., Zhu, X., Zheng, Z., et al. (2017). The individual identification method of wireless device based on dimensionality reduction and machine learning. Journal of Supercomputing, 5, $1-18$.

4. Li, J., \& Ying, Y. (2018). A method to improve the robustness of gas turbine gas-path fault diagnosis against sensor faults. IEEE Transactions on Reliability., 67(1), 3-12.

5. Min, J., Xuemai, G., Qing, G., Wei, X., \& Naitong, Z. (2016). Broadband hybrid satellite-terrestrial communication systems based on cognitive radio toward 5G. IEEE Wireless Communications, 23(6), 96-106.

6. Min, J., Xin, L., Zhisheng, Y., Qing, G., \& Xuemai, G. (2016). Joint cooperative spectrum sensing and spectrum opportunity for satellite cluster communication networks. Ad Hoc Networks, 58(C), 231-238.

7. Min, J., Xin, L., Xuemai, G., \& Qing, G. (2017). Joint cooperative spectrum sensing and channel selection optimization for satellite communication systems based on cognitive radio. International Journal of Satellite Communications and Networking, 35(2), 139-150.

8. Min, J., Zhisheng, Y., Qing, G., Gongliang, L., \& Xuemai, G. (2018). Downlink design for spectrum efficient iot network. IEEE Internet of Things Journal, 5(5), 3397-3404.

9. Dumitrescu, A., Popescu, D., Popescu, C. M., \& Petrescu, L. (2017). Classification based on the anatomo-fractal dimension in biology. In 21st international conference on system theory, control and computing (ICSTCC) (pp. 465-470).

10. Ying, Y. (2016). Study on gas turbine engine fault diagnostic approach with a hybrid of gray relation theory and gas-path analysis. Advances in Mechanical Engineering, 8(1), 1-14.

11. Zhang, Y.-D., Chen, X.-Q., Zhan, T.-M., Jiao, Z.-Q., Sun, Y., Chen, Z.-M., et al. (2016). Fractal dimension estimation for developing pathological brain detection system Based on Minkowski-Bouligand method. IEEE Access, 4, 5937-5947.

12. Sanchez, J. P. A., Alegria, O. C., Rodriguez, M. V., Abeyro, J. A. L. C., Almaraz, J. R. M., \& Gonzalez, A. D. (2017). Detection of ULF geomagnetic anomalies associated to seismic activity using EMD method and fractal dimension theory. IEEE Latin America Transactions, 15(2), 197-205.

13. Ji, L., Cao, Y., Ying, Y., \& Li, S. (2016). A rolling element bearing fault diagnosis approach based on multifractal theory and gray relation theory. PLOS ONE, 11(12), e0167587.

14. Ivanovici, M., \& Richard, N. (2011). Fractal dimension of color fractal images. Image Processing, 20(1), 227-235.

15. Ivanovici, M., \& Richard, N. (2011). Fractal dimension of color fractal images. Image Processing, 18(3), 31-35.

16. Jia, M., Yin, Z., Guo, Q., Liu, G., \& Gu, X. (2018). Toward improved offloading efficiency of data transmission in the IoTcloud by leveraging secure truncating OFDM. IEEE Internet of Things Journal. https://doi.org/10.1109/JIOT.2018.2875743.

17. Jia, M., Li, D., Yin, Z., Guo, Q., \& Gu, X. (2018). High spectral efficiency secure communications with non-orthogonal physical and multiple access layers. IEEE Internet of Things Journal, 99, 1-8. https://doi.org/10.1109/JIOT.2018.2851069.

18. Prieto, M. D., Espinosa, A. G., Ruiz, J.-R. R., et al. (2011). Feature extraction of demagnetization faults in permanent- 
magnet synchronous motors based on box-counting fractal dimension. Industrial Electronics, 58(5), 1594-1605.

19. Aklilu, G., \& Pitts, J. M. (2005). Multi-scaling chaotic map suitable for modelling aggregate TCP/IP and MPEG4 traffic streams. Electronics Letters, 41(12), 695-696.

20. Ding, K., Fang, X., Zhang, W., et al. (2012). Target identification of acoustic signals based on multifractal analysis and support vector machine. Journal of Ordnance Engineering, 33(12), $1521-1526$.

21. Yang, D., \& Wang, S. (2004). 1/f noise theory and its application to signal processing. Journal of Heilongjiang Institute of Technology, 18(3), 31-35.

22. Wu, J., \& Xu, C. (1996). The diffusion limited aggregation model and summariza-tion of fractal growth. Journal of Shandong University of Technology, 26(A09), 403-410.

23. Yu, B. (2003). Analysis of heat and mass transfer in fractal media. Journal of Engineering Thermophysics, 24(3), 481-483.

24. Chen, X., Li, J., Han, H., \& Ying, Y. (2018). Improving the signal subtle feature extraction performance based on dual improved fractal box dimension eigenvectors. Royal Society Open Science, 5.2(5), 1-12.

25. Zhang, X., Chen, W., Sun, Y., et al. (2007). The overview of fractal theory and its application in manufacturing system. Manufacturing Automation, 29(3), 4-9.

26. Han, H., Li*, J., \& Chen, X. (2018). The individual identification method of wireless device based on a robust dimensionality reduction model of hybrid feature information. Mobile Networks and Applications, 23(4), 709-716.

27. Zeng, R., Zhang, S., Zeng, R., Shen, H., \& Zhang, L. (2017). A method of fault detection on diesel engine based on EMD-fractal dimension and fuzzy C-mean clustering algorithm. In 29th Chinese control and decision conference (CCDC) (pp. 7679-7683).

28. Li, J., \& Ying, Y. (2014). Individual radiation source identification based on fractal box dimension. In IEEE 2014 2nd international conference on systems and informatics (ICSAI) ( $\mathrm{pp}$. 676-681).

29. Li, J., Ying, Y., Lou, X., et al. (2018). Integrated energy system optimization based on standardized matrix modeling method. Applied Sciences, 8(12), 2372-2391.

30. Ying, Y., Li, J., Chen, Z., \& Guo, J. (2017). Study on rolling bearing on-line reliability analysis based on vibration information processing. Computers and Electrical Engineering, 11(28), 1-10.

31. Tang, B., Tu, Y., Zhang, S., et al. (2018). Digital signal modulation classification with data augmentation using generative adversarial nets in cognitive radio networks. IEEE Access, 6, $15713-15722$.

32. Ma, X., Wang, T., Lin, Y., et al. (2018). Parallel iterative intercarrier interference cancellation in underwater acoustic orthogonal frequency division multiplexing. Wireless Personal Communications, 5, 1-14.

Publisher's Note Springer Nature remains neutral with regard to jurisdictional claims in published maps and institutional affiliations.

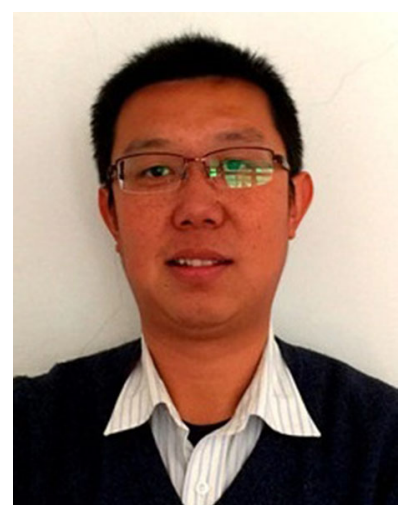

Zhen Zhang received the B.S. degree in electronical information engineering from Harbin Engineering University in 2003 and got M.S. degrees in communication engineering from Shanghai Maritime University. $\mathrm{He}$ is currently pursuing the Ph.D. degree in Harbin Engineering University with the Department of Information and Communication Engineering. His main studying area is signal analysis, including radio frequency fingerprint identification, pattern recognition, machine learning and deep learning.

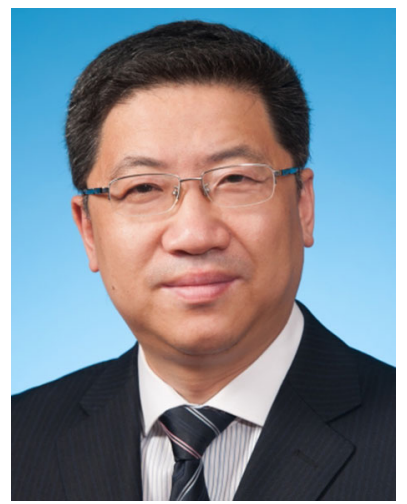

Yibing Li (M'09) received the B.S., M.S., and Ph.D. degrees from the Harbin Marine Engineering College, Harbin Engineering University Heilongjiang, China, in 1989, 1997, and 2003, respectively. He has been a Teacher with the Harbin Engineering University of China since 1989, and became a Professor in 2004. From 2007 to 2008, he was with the Electronic Engineering Laboratory, The University of Hong Kong, as a Visiting Scholar. He is currently a Professor with the Department of Information and Communication Engineering, Harbin Engineering University. He is a Senior Member of the China Institute of Communications and the China Computer Federation.

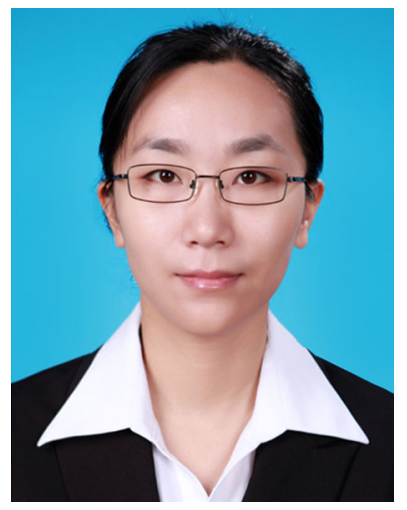

Lin Qi received the B.S., M.S. and Ph.D. degrees in Communication Engineering, Signal and Information Processing, and Communication and Information System from Harbin Engineering University, China in 2002, 2005 and 2011, respectively. From 2002 to 2005, she was an Assistant Professor of College of Information and Communication Engineering at Harbin Engineering University. Since 2007, she has been a Lecturer of College of Information and Communication Engineering at Harbin Engineering University. She also had been working at the Institute of Materials and Systems for Sustainability of Nagoya University from 2015 to 2016 as a visiting scholar. Her current research interests include, Wideband Digital Communication System, SDR, and Communication Signal Processing. 